\title{
Nanodroplet-Benzalkonium Chloride Formulation Demonstrates In Vitro and Ex-Vivo Broad-Spectrum Antiviral Activity Against SARS-CoV-2 and other Enveloped Viruses
}

Jessie Pannu

Bluewillow Biologics

Susan Ciotti

Bluewillow Biologics

Shyamala Ganesan

Bluewillow Biologics https://orcid.org/0000-0002-1021-3670

George Arida

Bluewillow Biologics

Chad Costley

BlueWillow Biologics

Ali Fattom ( $\square$ ali.fattom@bluewillow.com )

BlueWillow Biologics

\section{Research note}

Keywords: Antiviral, Nanoemulsion, Nanodroplets, Human Skin, and SARS-CoV-2

Posted Date: March 31st, 2021

DOI: https://doi.org/10.21203/rs.3.rs-362864/v1

License: (c) (1) This work is licensed under a Creative Commons Attribution 4.0 International License.

Read Full License 


\section{Abstract}

Objective: The Covid-19 pandemic has highlighted the importance of aerosolized droplets inhaled into the nose in the transmission of respiratory viral disease. Inactivating pathogenic viruses at the nasal port of entry may reduce viral loads, thereby reducing infection, transmission and spread. In this communication, we demonstrate safe and broad anti-viral activity of oil-in-water nanoemulsion (nanodroplet) formulation containing the potent antiseptic $0.13 \%$ Benzalkonium Chloride (NE-BZK).

Results: We have demonstrated that NE-BZK exhibits broad-spectrum, long-lasting antiviral activity with $>99.9 \%$ in vitro killing of enveloped viruses including SARS-CoV-2, human coronavirus, RSV, and influenza B. In vitro and ex-vivo studies demonstrated continued killing of $>99.99 \%$ of human coronavirus with diluted NE-BZK and persistent for 8 hours post application, respectively. The repeated application of NEBZK, twice daily for 2 weeks into rabbit nostrils demonstrated its safety with no nasal irritation. These findings demonstrate that formulating BZK into the proprietary nanodroplets offers a safe and effective antiviral and a significant addition to strategies to combat the spread of respiratory viral infectious diseases.

\section{Introduction}

The nasal cavity is a primary port of entry for bacterial and viral respiratory pathogens. Recently published studies on SARS-CoV-2 highlight the prominent role of nasal epithelium in initial infection, viral replication, colonization, and transmission. ${ }^{1-3}$ Other highly contagious and pathogenic viruses such as influenza, respiratory syncytial virus (RSV) and common human coronaviruses also enter through the nasal mucosa, causing upper and lower respiratory tract infection. ${ }^{4-6}$ Increased viral load in the nasal mucosa was shown to result in viral aspiration into the lungs ${ }^{7}$ causing severe lower respiratory tract disease with high morbidity and mortality. The current Covid-19 pandemic has raised the urgent need for a broad-spectrum antiviral nasal antiseptic that can prevent, or reduce viral colonization, infection, and transmission of pathogenic respiratory viruses.

The CDC has reported varied antiviral activity of the widely used skin antiseptic AQ-BZK, including that it can be ineffective against coronavirus. ${ }^{8,9} \mathrm{NE}-\mathrm{BZK}$ is a proprietary antiseptic which incorporates the active ingredient benzalkonium chloride $(0.13 \%$ BZK) in oil-in-water nanodroplets. Nanodroplets are $350 \mathrm{~nm}$ in size with positively charged surfaces, that repel each other and keeps the active ingredient BZK from inactivating through crystallization. ${ }^{10}$ The surface of enveloped viruses (e.g., SARS-CoV-2, influenza, RSV) are negatively charged. ${ }^{11}$ Therefore, the positively charged nanodroplets in NE-BZK are attracted to these viruses, delivering the antiseptic payload directly to the surface of the pathogen where splitting and killing of the virus occurs. Nanoemulsions with different API has been used to produce an inactivated split virus preparation including RSV, HSV, Flu, and Zika viruses (Unpublished data). Here we report data from in vitro and ex vivo antiviral testing of the new formulation NE-BZK along with demonstration of in vivo safety of its intranasal application in rabbits. 


\section{Materials And Methods}

Nanoemulsion Formulation with BZK: The nanoemulsion formulation was prepared with $0.13 \%$ benzalkonium chloride (BZK) as the active ingredient. ${ }^{12}$ BZK, a quaternary ammonium compound, was chosen due to its inherent antimicrobial activity and is currently use in numerous nasal spray and skin antiseptic products. In NE-BZK, the BZK resides at the interface between the oil and water phases of the nanodroplets with the hydrophobic tail distributed in the oil core and the polar cationic head group residing at the oil-water interface.

Virus Strains Used for Demonstration of Antiviral Activity with NE-BZK: Broad spectrum antiviral activity of NE-BZK was tested using the following viral isolates: SARS-CoV-2 Victoria/1/2020 strain (Public Health England (PHE), Porton Down, Salisbury, UK); Human coronavirus 229E (ATCC: VR-740); Influenza B (VR-1931); and Respiratory Syncytial Virus (BlueWillow Biologics in-house strain: NBL-14-001-2UC). The virus growth media was either Minimum Essential Medium - Eagle with Earle's BSS (MEM Eagle EBSS) from Lonza (Rochester, NY) or Dulbecco's Modified Eagle's Medium (DMEM) from Corning Inc (Corning, NY). The cells used in the virus studies were obtained from ATTC (Manassas, VA): Vero E6 cells (ATCC\# CRL 1586) for SARS-CoV2, MRC-5 cells for HCoV229E (CCL-171 ATCC), MDCK cells for Influenza B (CCL34 ATCC) and Vero cells for RSV (CCL-81 ATCC).

\section{In Vitro Determination of Antiviral Activity}

Using the time kill procedures described in the Standard Guide ASTM E1052-11, the antiviral activity of the NE-BZK was assessed by inoculating the formulation with a suspension of viral particles (final concentration of 1.5-3.1 $\left.\times 10^{6} \mathrm{PFU} / \mathrm{ml}\right) .{ }^{13}$ At a predetermined exposure time, an aliquot was taken and neutralized to remove residual effect of NE-BZK by diluting at 1:100 dilution in cell growth media containing $1-2 \%$ FBS. A comparative inactivation of HCoV 229E by NE-BZK and AQ-BZK was also evaluated by using the test samples at full strength, 1/10 dilution, and1/20 dilution.Virus control with virus alone and toxicity control with test sample alone was also included. Concentration of active virus particles was determined quantitively by plaque or $T_{C I D}$ assay. $T C I D_{50}$ was calculated by the Karber

method. ${ }^{14}$ Number of PFU recovered from the test sample was converted into $\log _{10}$ format and compared to an initial starting concentration to determine a log reduction.

\section{Ex Vivo Persistence of Antiviral Activity on Human Cadaver Skin Following Application of NE-BZK}

Cryopreserved, dermatomed human cadaver abdominal skin from Caucasian donors was obtained from Science Care organ donor bank (Phoenix, AZ). The permeation and retention of each antiseptic preparation in human skin was determined using the ex vivo permeation technique described by Franz. ${ }^{15}$ In brief, human skin was placed onto a Franz diffusion cell chamber and secured. The skin was maintained at a temperature and humidity that match typical in vivo conditions. Human skin was dosed with either the nanoemulsion antiseptic (NE-BZK) or aqueous BZK solution (AQ-BZK) applied at a single dose of $100 \mathrm{~mL} / \mathrm{cm}^{2}$. At either 4 or 8 hours post topical application, $10 \mu \mathrm{L}$ of viral particles in suspension 
(final concentration of 1-3 $\times 10^{5} \mathrm{PFU} / \mathrm{ml}$ ) was applied to the skin surface for a contact time of 20 minutes. The skin surface was washed with growth media, pooled and neutralized to remove residual effect of the test formulation by diluting at 1:100 in growth media. Concentration of virus particles was determined quantitively by plaque or $\mathrm{TCID}_{50}$ assay.

\section{In Vivo Rabbit Safety and Toxicology Study}

The safety of NE-BZK was evaluated in New Zealand white rabbits following bi-dose nasal swab application for two weeks. The exploratory study was performed at IITRI in accordance with protocols approved by the animal care and use committee. Following acclimation, the animals were randomly assigned to either naïve control or NE-BZK treatment groups with 2 /sex in control group and 4/sex in NEBZK group. The NE-BZK was applied to the inner nostril and nasal mucosa using a puritan foam tip. Two hours post last dose application and two days later, blood was collected by bleeding the central ear artery. Serum was analyzed by HPLC for BZK. The animals were sacrificed 2 days post last administration by an overdose of sodium pentobarbital ( $150-200 \mathrm{mg} / \mathrm{kg}$ by intravenous administration) and nasal cavity was examined macroscopically and scored for erythema and edema by Draize method of scoring for dermal irritation. ${ }^{16}$

\section{Analysis of Serum Samples for BZK}

Rabbit serum samples collected at two hours and two days post NE-BZK application was analyzed using qualified reverse phase HPLC method. The samples were extracted with acetonitrile and filtered before injecting into column. The samples were run on Phenomenex Luna $5 \mu$ column, using $0.04 \mathrm{M}$ sodium acetate in acetonitrile as mobile phase. The samples were run at a flow rate of $2 \mathrm{~mL} /$ minute with $100 \mu \mathrm{L}$ injection volume. The peaks were detected using $254 \mathrm{~nm}$ wavelength. The analytical assay detection limit was $0.094 \mu \mathrm{g} / \mathrm{mL}$.

\section{Results}

\section{Broad Spectrum Antimicrobial Activity of Nanoemulsion with BZK Formulation}

Since the well-known antiseptic BZK in standard aqueous solutions has demonstrated varied activity against viruses and bacteria, including human coronavirus, we formulated BZK in oil-in-water nanoemulsion and evaluated the broad-spectrum antiviral activity of NE-BZK. Data presented in Fig. 1 shows that NE-BZK inactivated $>99.9 \%$ of all tested enveloped viruses, including SARS-CoV-2. The inactivation of these viruses was achieved within 5 minutes of mixing with the nanoemulsion. The results of these studies demonstrated that NE-BZK has a sustained and consistent broad-spectrum antiviral activity against multiple respiratory pathogens, including SARS-CoV-2, Influenza B, and RSV. 
In Vitro Efficacy of Aqueous BZK in Comparison to BZK Formulated in Nanoemulsion against Human Coronavirus (HCoV229E)

To demonstrate the superior antiviral activity of NE-BZK despite dilution, which is inherent in skin or mucosal application in vivo, we compared serial dilutions of NE-BZK and aqueous BZK in the in vitro inactivation test against human coronavirus. Three different concentrations including full-strength $(0.13 \%$ BZK), $1 / 10$ dilution ( $0.013 \%$ BZK) and $1 / 20$ dilution ( $0.0065 \%$ BZK), was tested against human coronavirus (HCoV229E) following five-minute exposure. Data presented in Table 1 demonstrate that even at highest tested dilution of $1 / 20$, the NE-BZK formulation continued to demonstrate $>99.99 \%$ killing while aqueous AQ-BZK antiviral activity was diminished with dilutions.

Table 1

In vitro log reduction of human coronavirus (HCoV 229E) treated for 5 minutes with NE$B Z K$ versus $A Q-B Z K$ at three dilution levels

\begin{tabular}{|lllll|}
\hline Product (\%BZK) & AQ-BZK & \multicolumn{3}{l|}{ NE-BZK } \\
\cline { 2 - 5 } & Log Reduction & \% Killing & Log Reduction & \% Killing \\
\hline Full Strength $(0.13 \%)$ & $>4.49$ & $>99.99$ & $>4.49$ & $>99.99$ \\
\hline 1/10 Dilution $(0.013 \%)$ & $>4.49$ & $>99.99$ & $>4.49$ & $>99.99$ \\
\hline 1/20 Dilution $(0.0065 \%)$ & $<1$ & $<10$ & $>4.49$ & $>99.99$ \\
\hline
\end{tabular}

\section{Durability of NE-BZK Efficacy in ex-vivo Human Cadaver Skin}

Following demonstration of in vitro activity against human coronavirus, an ex-vivo time-kill study was performed following pre-treatment of human cadaver skin with NE-BZK $(0.13 \%$ BZK) or AQ-BZK for 4 and 8 hours. As presented in Table 2, NE-BZK achieved $>4.7 \mathrm{log}$ killing of HCoV 229E at both the 4- and 8-hour time points, while AQ-BZK exhibited only 1.5 log killing at 4 hours and no detectable activity at 8 hours. These data suggest that NE-BZK could maintain its antiviral activity for a longer time due to stability of this formulation compared to AQ-BZK formulations.

Table 2

Ex vivo log reduction of HCoV 229E by Nanoemulsion Antiseptic (NE-BZK) versus an AQ-BZK at 1:10 dilutions after 4 and 8 hours post application on human skin.

\begin{tabular}{|lll|}
\hline Product & 4 Hours & 8-Hours \\
\cline { 2 - 3 } 1/10 Dilution (\%BZK) & $\begin{array}{l}\text { Average Log Kill* } \\
\text { (PFU/skin sample) }\end{array}$ & $\begin{array}{l}\text { Average Log Kill* } \\
\text { (PFU/skin sample) }\end{array}$ \\
\hline NE-BZK $(0.013 \%$ BZK) & $>4.7$ & $>4.7$ \\
\hline AQ-BZK $(0.013 \%)$ & 1.5 & $<7$ * \\
\hline *Virus killing is calculated based on recovery from WFI control samples (5.70 Logs per skin sample). \\
\hline
\end{tabular}


In-vivo Safety and Non-Irritating Properties of NE-BZK Product Applied to Nasal Skin Surfaces

Demonstration of broad-spectrum anti-viral activity of NE-BZK makes application to the nasal cavity an option to reduce viral load, prevent colonization and lessen disease progression. Therefore, we studied the safety of multiple daily nasal administration of NE-BZK in New Zealand white rabbits. Following 2week bi-dose intranasal application of NE-BZK, none of the animals in the treatment group $(\mathrm{N}=8)$ exhibited any signs of erythema or edema and were indistinguishable from the naïve group $(N=4)$. Furthermore, BZK values in serum samples collected 2 hours and 2 days post application of the final dose were below the limit of detection of BZK $(<0.094 \mu \mathrm{g} / \mathrm{ml})$ demonstrating that this treatment localizes in the nasal cavity and does not reach the blood stream.

\section{Discussion}

The nasal cavity is the primary port of entry for almost all respiratory disease-causing viruses. ${ }^{1-3} \mathrm{An}$ effective nasal antiseptic that inactivates the virus at the port of entry will be essential in preventing infection, colonization, and transmission. ${ }^{7}$

Benzalkonium chloride (BZK), a well-known effective and safe antiseptic, has demonstrated variable antiviral activity in typical aqueous formulations. In order to enhance antiviral activity and facilitate safe delivery in the nasal cavity, we formulated $0.13 \%$ BZK as the main cationic surfactant component in a new oil-in-water nanoemulsion (NE-BZK). Our data demonstrate enhanced antiviral activity of NE-BZK versus commonly available aqueous $0.13 \%$ BZK formulations.

The timeline of SARS-CoV-2 infection indicates high viral load in infected individuals during the incubation period, and 5-7 days post symptom onset results in the increased infectivity $7,18,19$ and has been shown to be the most significant predictor of severe COVID-19 disease including death. ${ }^{20}$ Therefore, intervention aimed at reducing nasal viral load in SARS-CoV-2 infected persons would likely not only increase the probability of survival but also likely reduce viral transmission to those in contact with the infected individual. A recent article by Frank et al shows the in vitro efficacy of povidone-iodine nasal antiseptic for rapid inactivation of SARS-CoV-2. However, this approach has major limitations including allergic potential, skin irritation and thyroid disease concerns. Povidone-iodine can become ineffective when diluting from available commercial $5 \%$ or $10 \%$ solutions and can be effective only up to 4 hours post application ${ }^{21}$. NE-BZK has an added advantage that safety has been previously demonstrated in humans through two different phase 1 clinical trials using the same nanoemulsion technology with a similar cationic surfactant. ${ }^{22,23}$ BZK at concentrations similar to those in NE-BZK has been used in human skin products since the 1940's and is currently marketed in numerous nasal products. ${ }^{24}$ Hirose et al has shown the survival of SARS-CoV-2 on skin surfaces for 9 hours ${ }^{25}$ which may require a more durable antiseptic activity. Our ex-vivo data has shown that antimicrobial effect of NE-BZK was seen even at 8hours post application, and even when diluting 20-fold, suggesting that application every 8 hours may be useful in protecting against disease. Our in vivo safety study in rabbits also demonstrated no safety issues with twice daily application of NE-BZK for two weeks with no systemic absorption. 
While newly diagnosed persons are sent home for quarantine without any recommended intervention, we believe that an antiviral NE-BZK antiseptic might serve as a tool for reducing viral loads in SARS-Cov-2 quarantined infected persons, potentially reducing the risk of disease progression, transmission to close contacts and households, and increasing survival.

\section{Conclusions}

NE-BZK is a safe, non-irritating nasal antiseptic, which may help in mitigating transmission of SARS-CoV2 and other respiratory viruses through nasal droplets at the port of entry.

\section{Limitations}

This study's limitation was that NE-BZK was not tested in animal models or human subjects as an antiviral nasal sanitizer to treat or prevent viral colonization and infections.

\section{Abbreviations}

SARS- CoV-2: Severe Acute Respiratory Syndrome Coronavirus -2; NE-BZK: Nanoemulsion with $0.13 \%$ Benzalkonium Chloride; RSV: Respiratory Syncytial Virus; CD: Centre for Disease Control; API: Active Pharmaceutical Ingredient; AQ-BZK: Aqueous solution of benzalkonium chloride; ATCC: American Type Culture Collection; MDCK: Madin- Darby Canine Kidney Cells; HCoV229E: Human Coronavirus 229E strain; FBS: Fetal Bovine Serum; MEM: Minimum Essential Medium; HPLC: High Pressure Liquid Chromatography.

\section{Declarations}

\section{Ethics Approval and Consent to Participate}

Rabbit study was conducted by IIT Research Institute in accordance with protocols approved by the animal care and use committee (IACUC Approval \# 20-049).

\section{Consent for Publication}

Not applicable

\section{Availability of Data and Materials}

All data generated or analyzed during this study are included in this article

\section{Competing Interests}

All authors are employees of BlueWillow Biologics

\section{Funding}


This research received no specific grant from any funding agency in the public, commercial, or not-forprofit sectors.

\section{Author's Contributions}

JP designed and performed the in vitro antiviral testing, analyzed data and revised manuscript draft; SC designed the study, prepared formulation and performed ex-vivo experiments; SG designed the rabbit study, analyzed data from rabbit study, wrote and revised manuscript draft; GA and CC revised manuscript draft; AF supervised the study and revised the manuscript. All authors read the manuscript, revised and accepted it.

\section{Acknowledgments}

We are grateful to Bassam Hallis, Head of Pre-Clinical Dev \& General Project Manager National Infection Service and Kevin Dyer, Senior Business Development Manager, for supporting the in vitro studies on SARS-CoV-2 that were conducted at the Public Health England (PHE), Porton Down facility, Salisbury SP4 OJG United Kingdom. We thank Sue Charlton for her excellent technical assistance in running the SARSCov-2 in vitro experiments at PHE.

We are thankful to Lakshman Caldera from BlueWilow Biologics for analyzing the serum samples from rabbit study. All of the authors whose names are listed are employees of BlueWillow Biologics.

\section{References}

1. He X, Lau EHY, Wu P, Deng X, Wang J, Hao X, Lau YC, Wong JY, Guan Y, Tan X, Mo X, Chen Y, Liao B, Chen W, Hu F, Zhang Q, Zhong M, Wu Y, Zhao L, Zhang F, Cowling BJ, Li F, Leung GM. Temporal dynamics in viral shedding and transmissibility of COVID-19. Nature Medicine. 2000; 26: 672-675.

2. Zou L, Ruan F, Huang M, Liang L, Huang H, Hong Z, Yu J, Kang M, Song Y, Xia J, Guo Q, Song T, He J, Yen HL, Peiris M, Wu J. SARS-CoV-2 viral load in upper respiratory specimens of infected patients. N Engl J Med. 2020; 382:1177-1179.

3. Wang W, Xu Y, Gao R, Lu R, Han K, Wu G, Tan W. Detection of SARS-CoV-2 in different types of clinical specimens. JAMA. 2020; 18:1843-1844.

4. Richard M., van den Brand J, Bestebroer, T, Lexmond P, de Meulder D, Fouchier RAM, Lowen AC, Herfst S. Influenza A viruses are transmitted via the air from the nasal respiratory epithelium of ferrets. Nature Communications. 2020; 11: 1-11.

5. Glezen WP, Taber LH, Frank AL, Kasel JA. Risk of primary infection and reinfection with respiratory syncytial virus. Am J Dis Child. 1986; 140(6):543-6.

6. Chilvers M, McKean M, Rutman A, Myint, B, Silverman M, O'Callaghan C. The effects of coronavirus on human nasal ciliated respiratory epithelium. European Respiratory Journal. 2001; 18: 965-970.

7. Hou YJ, Okuda K, Edwards CE, Martinez DR, Asakura T, Dinnon KH 3rd, Kato T, Lee RE, Yount BL, Mascenik TM, Chen G, Olivier KN, Ghio A, Tse LV, Leist SR, Gralinski LE, Schäfer A, Dang H, Gilmore R, 
Nakano S, Sun L, Fulcher ML, Livraghi-Butrico A, Nicely NI, Cameron M, Cameron C, Kelvin DJ, de Silva A, Margolis DM, Markmann A, Bartelt L, Zumwalt R, Martinez FJ, Salvatore SP, Borczuk A, Tata PR, Sontake V, Kimple A, Jaspers I, O'Neal WK, Randell SH, Boucher RC, Baric RS. SARS-CoV-2 Reverse Genetics Reveals a Variable Infection Gradient in the Respiratory Tract. Cell. 2020; Jul 23;182(2):429-446.e14.

8. CDC Guidance document on Hand Hygiene recommendations- Guidance for Healthcare Providers about Hand Hygiene and COVID 19.

9. Wood A, Payne D. The action of three antiseptics/disinfectants against enveloped and nonenveloped viruses. J Hosp Infect. 1998;38(4):283-95

10. Thomas L, Russell A, Maillard, J. Antimicrobial activity of chlorhexidine diacetate and benzalkonium chloride against Pseudomonas aeruginosa and its response to biocide residues. Journal of Applied Microbiology. 2005; 98: 533-543.

11. Zanin M, Baviskar P, Webster R, Webby R. The interaction between respiratory pathogens and mucus. Cell Host Microbe. 2016; 19(2): 159-168

12. Makidon PE, Bielinska AU, Nigavekar SS, Janczak KW, Knowlton J, Scott AJ, Mank N, Cao Z, Rathinavelu S, Beer MR, Wilkinson JE, Blanco LP, Landers JJ, Baker JR Jr. Pre-clinical evaluation of a novel nanoemulsion-based hepatitis B mucosal vaccine. PLoS One. 2008; 13;3(8): e2954.

13. ASTM E1052-11, Standard Test method to assess the activity of microbicides against viruses in suspension, ASTM International, West Conshohocken, PA, 2020, astm.org.

14. Lambert F, Jacomy H, Marceau G, Talbot P. Titration of human coronaviruses, HCoV-229E and HCoVOC43, by an indirect immunoperoxidase assay, p. 93-102. In Cavanagh D (ed), Methods in Molecular Biology, 2020; vol. 454: SARS- and Other Coronaviruses, Humana Press, New York, NY.

15. Franz, TJ. Percutaneous absorption: on the relevance of in vitro J. Invest. Dermatol. 1975;64(3):190195.

16. Draize JH, Woodward G, Calvery HO. Methods for the Study of Irritation and Toxicity of Substances Applied Topically to the Skin and Mucous Membranes. J Pharmacology Experi Thera. 1994; 82 (3):377-390.

17. Lauer SA, Grantz KH, Bi Q, Jones FK, Zheng Q, Meredith HR, Azman AS, Reich NG, Lessler J. The Incubation Period of Coronavirus Disease 2019 (COVID-19) From Publicly Reported Confirmed Cases: Estimation and Application. Ann Intern Med. 2020; 172(9):577-582.

18. Bar-On YM, Flamholz A, Phillips R, Milo R. SARS-CoV-2 (COVID-19) by the numbers. Elife. 2020; Apr 2;9: e57309.

19. Pujadas E, Chaudhry F, McBride R, Richter F, Zhao S, Wajnberg A, Nadkarni G, Glicksberg BS, Houldsworth J, Cordon-Cardo C. SARS-CoV-2 viral load predicts COVID-19 mortality. Lancet Respir Med. 2020; 8(9): e70.

20. Frank S, Brown SM, Capriotti JA, Westover JB, Pelletier JS, Tessema B. In Vitro Efficacy of a Povidone-lodine Nasal Antiseptic for Rapid Inactivation of SARS-CoV-2. JAMA Otolaryngol Head Neck Surg. 2020; e203053. 
21. Stanberry LR, Simon JK, Johnson C, Robinson PL, Morry J, Flack MR, Gracon S, Myc A, Hamouda T, Baker JR Jr. Safety and immunogenicity of a novel nanoemulsion mucosal adjuvant W805EC combined with approved seasonal influenza antigens. Vaccine. 2012; 30(2):307-16

22. Kircik L, Jones TM, Jarratt M, Flack MR, ljzerman M, Ciotti S, Sutcliffe J, Boivin G, Stanberry LR, Baker JR. NB-001 Study Group. Treatment with a novel topical nanoemulsion (NB-001) speeds time to healing of recurrent cold sores. J Drugs Dermatol. 2012;11(8):970-7.

23. Bondurant S, McKinney T, Bondurant L, Fitzpatrick L. Evaluation of a benzalkonium chloride hand sanitizer in reducing transient Staphylococcus aureus bacterial skin contamination in health care workers. Am J Infect Control. 2019; 48(5):522-526.

24. Ryohei Hirose, Hiroshi Ikegaya, Yuji Naito, Naoto Watanabe, Takuma Yoshida, Risa Bandou, Tomo Daidoji, Yoshito Itoh, Takaaki Nakaya. Survival of SARS-CoV-2 and influenza virus on the human skin: Importance of hand hygiene in COVID-19, Clinical Infectious Diseases. 2020; ciaa1517.

\section{Figures}

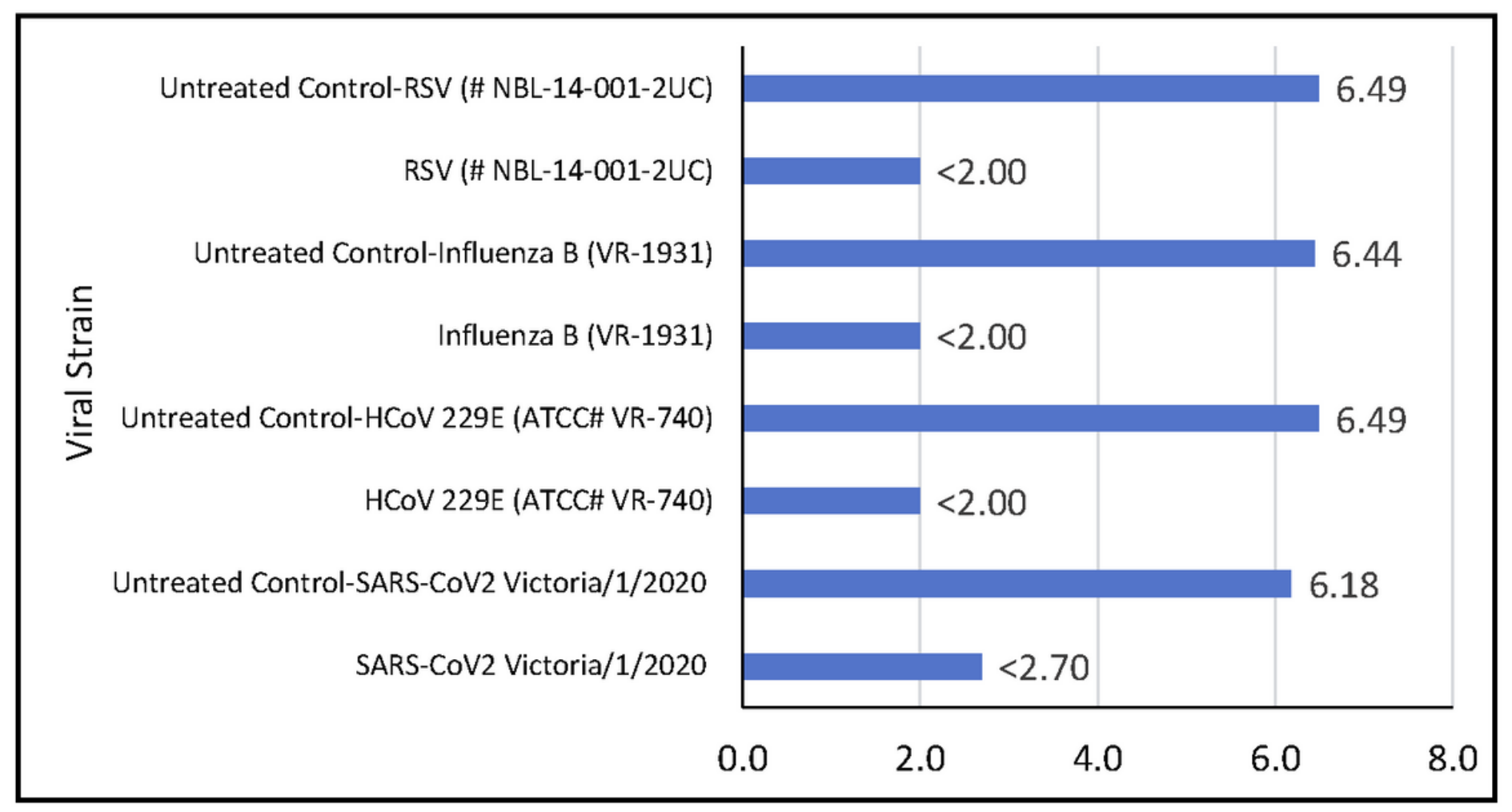

\section{Figure 1}

Log Reduction of Enveloped Viruses when Treated with Nanoemulsion Formulated with BZK The antiviral effect of NE BZK was studied by inoculating the formulation with viral particles Following five minute exposure, all the tested enveloped viruses demonstrated 999 killing, which was confirmed by plaque or TCID 50 assay NOTE Virus killing was calculated based on recovery from virus control samples (SARS 
CoV $2618 \mathrm{Log}$ PFU/mL, HCoV 229 E= 649 Log PFU/mL, Influenza B 644 Log PFU/mL, RSV 649 Log $\mathrm{PFU} / \mathrm{mL}$ ) Toxicity control indicated that 1100 or 1500 dilution of treated sample was required to remove the toxicity effect to host cells Therefore, lower limit of detection (was 2270 Logs PFU/mL

\section{Supplementary Files}

This is a list of supplementary files associated with this preprint. Click to download.

- ARRIVEChecklist.pdf 